\title{
EFFETS DE L'EXTRAIT AU CYCLOHEXANE DE BIDENS PILOSA L. (ASTERACEAE) SUR LA MOTRICITE INTESTINALE DE RAT
}

\author{
Dimo $^{1}$ Théophile, A. Rakotonirina ${ }^{2}$, Kamgang R. ${ }^{1}$, Tankwa $^{1}$, T. Roger et \\ K. Wanda ${ }^{1}$ Germain Jean Magloire \\ ${ }^{1}$ Laboratoire de Physiologie animale, Université de Yaoundé I - B.P. 812 Yaoundé, Cameroun. \\ ${ }^{2}$ Département des Sciences Biologiques - Ecole Normale Supérieure de Yaoundé, Cameroun.
}

\begin{abstract}
RÉSUMÉ: Nous avons examiné in vitro, l'effets de l'extrait au cyclohexane des feuilles de Bidens pilosa sur l'activité contractile de fragments d'iléon de rat. Sur les contractions spontanées du muscle lisse iléal ces extraits de Bidens pilosa ont des effets stimulateurs dose-dépendante sur le tonus et l'amplitude. La stimulation maximale est observée à partir de $160 \mu \mathrm{g} / \mathrm{mL}$ d'extrait, avec un taux de variation de contraction de $1027 \%$. L'atropine réduit de $77 \%$ les effets spasmogènes de Bidens pilosa et de $94 \%$ celui de l'acétylcholine. En milieu dépourvu de Ca ${ }^{2+}$, l'accroissement de la contraction iléale induit par $160 \mu \mathrm{g} / \mathrm{mL}$ d'extrait n'est que de $400 \%$. Par ailleurs, la nifédipine inhibe totalement l'action spasmogène de cet extrait. Ces résultats font penser que l'extrait au cyclohexane des feuilles de Bidens pilosa stimulerait les contractions du muscle lisse iléal; ceci par l'intermédiaire des récepteurs muscariniques dont l'activation augmenterait la mobilisation du $\mathrm{Ca}^{2+}$ d'origine aussi bien extracellulaire qu'intracellulaire.
\end{abstract}

Mots clés : Bidens pilosa ; iléon; motricité ; rat.

ABSTRACT: Effects of cyclohexane extract of Bidens pilosa $L$. on rat intestinal motility. $-\underline{\text { In vitro, }}$ we have studied the effects of the cyclohexane extract of Bidens pilosa leaves (B.p.) on the rat ileum smooth muscle motility. B.p. has showed dose-dependent stimulators effects on both tonicity and amplitude of the spontaneous contractions. With $160 \mu \mathrm{g} / \mathrm{mL}$ of extract maximal stimulation was obtained. With that dose, the rate of variation was $1027 \%$. Atropine reduced by $77 \%$ the spasmogenic effects of B.p. and by $94 \%$ the acetylcholine effects. In $\mathrm{Ca}^{2+}$ free solution, stimulation contraction by $160 \mu \mathrm{g} / \mathrm{mL}$ of the extract was reduced to $400 \%$, while Nifedipine completely inhibited the spasmogenic effects of the extract. These results suggest that the cyclohexane extract of B.p. could stimulate ileal smooth muscle contractions through muscarinic receptors which increase $\mathrm{Ca}^{2+}$ mobilization from both extra- and intracellular milieu.

Key words : Bidens pilosa ; ileum ; motility; rat.

\section{INTRODUCTION}

De multiples affections s'accompagnent de troubles de la motricité digestive. Ces troubles fonctionnels digestifs représentent le motif de consultation le plus fréquent de la pathologie digestive (Cloarec et al., 1989). Bidens pilosa L. (Asteraceae) est très utilisée par les tradipraticiens des zones tropicales pour faciliter les accouchements, soigner les affections grippales, les otites, les ophtalmies, les diarrhées et la dysenterie (Bouquet, 1969, Keharo et Gadam, 1973, Adjanohoun et al., 1988). Cette plante possède également des propriétés antihypertensives (Dimo et al., 1999). Des analyses phytochimiques des extraits des feuilles de Bidens pilosa ont révélé la présence des glycosides et des chalcones (Hoffmann et Holzi, 1989), des terpènes et des huiles essentielles (Amvam Zollo et al.,1995), des composés acétyléniques et des flavonoïdes (Brandao et al., 1997). Ces auteurs ont montré que ces composés présentent des activités antimicrobiennes et antiinflammatoires.

Suite aux propriétés vasorelaxantes de Bidens pilosa observées par Dimo et al. (1998), nous examinons dans ce travail, les effets de l'extrait au cyclohexane des feuilles de cette plante sur l'activité contractile de fragments isolés d'iléons de rat. 


\section{Matériel et méthode. L'extrait de Bidens pilosa}

Les feuilles fraîches de Bidens pilosa récoltées aux environs du campus de l'université de Yaoundé I sont séchées au soleil puis broyées en une poudre fine. $2 \mathrm{~kg}$ de cette poudre sont mis à macérer dans $6 \mathrm{~L}$ de méthanol, à la température ambiante, pendant 7 jours. Filtré, le macérât est évaporé sous vide et sous pression réduite. $18 \mathrm{~g}$ de l'extrait ainsi obtenu sont épuisés dans $3 \mathrm{~L}$ de cyclohexane. La phase cyclohexanique surnageante est filtrée et concentrée sous pression réduite et sous vide, donnant ainsi $7 \mathrm{~g}$ d'extrait au cyclohexane de $B$. pilosa.

$1 \mathrm{~g}$ de l'extrait au cyclohexane des feuilles de B. pilosa est dissout dans $1 \mathrm{~mL}$ de diméthyle sulphoxide (DMSO) et repris dans $49 \mathrm{~mL}$ d'eau distillée, donnant ainsi une solution à $20 \mathrm{mg} / \mathrm{mL}$. Cet extrait est dilué au $10^{\text {ème }}$ avec le liquide physiologique au moment de l'utilisation.

\section{Evaluation de l'activité contractile du muscle lisse}

Les rats $(150-200 \mathrm{~g})$ sont tués par dislocation cervicale. La cavité abdominale est ouverte et des fragments d'iléon de 1 à $2 \mathrm{~cm}$ chacun sont prélevés et débarrés du tissu conjonctif. Les fragments d'iléon sont conservés pendant les essais à $37^{\circ} \mathrm{C}$ dans le liquide physiologique de Tyrode oxygéné, de composition (mM): $\mathrm{CaCl}_{2}$ (1.80), $\mathrm{KCl}$ (3.60), $\mathrm{NaCl}$ (136.75), $\mathrm{NaH}_{2} \mathrm{PO}_{4}(0.42), \mathrm{MgCl}_{2}$ (1.06), $\mathrm{NaHCO}_{3}$ (11.9), glucose (5.5). Chaque fragment d'iléon est monté dans une cuve à organe isolé et relié à un transducteur isotonique (type Ugo Basile type 7006). Les contractions sont enregistrées sur dynographe (Ugo Basile type GEMINI 7070 multivitesse à 2 pistes). Un volume de l'extrait au cyclohexane de B. pilosa $(2 \mathrm{mg} / \mathrm{mL})$ est ajouté au milieu d'incubation et les effets sont observés. Après 2 à 3 lavages au Tyrode et stabilisation pendant $30 \mathrm{~min}$, une dose supérieure d'extrait est ajoutée dans la chambre à organes. Différentes doses d'extrait sont évaluées sur l'activité contractile du muscle lisse iléal; comparée à celle de l'acétylcholine. Les effets de $B$. pilosa sont aussi examinés en milieu physiologique dépourvu de calcium. Sur des fragments d'iléon traités au préalable avec de l'atropine nous avons comparé les effets de $B$. pilosa et de l'acétylcholine.

\section{Traitement statistique des résultats}

Les moyennes arithmétiques (m) des valeurs individuelles affectées de l'erreur standard sur la moyenne ( \pm ESM) sont analysées par le test non paramétrique U de Mann et Whitney.

\section{RÉSULTATS}

L'extrait au cyclohexane de B. pilosa modifie aussi bien la tonus de base que l'amplitude des contractions du muscle lisse iléal (fig. 1). L'extrait de B. pilosa aux concentrations de 10 à $320 \mathrm{mg} / \mathrm{mL}$ provoque des augmentations dosedépendante de la contraction spontanée du muscle lisse iléal de rat : de 112 à $1097 \%$ (tableau 1). L'acétylcholine aux doses de $0.5-8 \mathrm{mg} / \mathrm{mL}$ induit également des augmentations de la contraction de l'iléon : 139 à $1046 \%$. Le traitement préalable du fragment d'iléon avec l'atropine $(0.2 \mathrm{mg} / \mathrm{mL})$ inhibe de $77 \%$ l'action contracturante de l'extrait de B. pilosa (160 mg/mL). L'atropine, à dose croissante, diminue jusqu'à $94 \%$ l'activité contractile induite par l'acétylcholine $(8 \mathrm{mg} / \mathrm{mL})$.

Tableau 1 : Effets de l'extrait au cyclohexane de Bidens pilosa sur le tonus de contraction spontanée du muscle

\begin{tabular}{l|cc}
\hline \multicolumn{1}{c|}{ Traitement } & Dose $(\mu \mathbf{g} / \mathbf{m L})$ & $\begin{array}{c}\text { \% variation de } \\
\text { la contraction }\end{array}$ \\
\hline Contrôle & - & - \\
Extrait & 10 & $112^{*}$ \\
& 20 & $504^{*}$ \\
& 40 & $604^{*}$ \\
& 80 & $892^{*}$ \\
& 160 & $1027^{*}$ \\
& 320 & $1097^{*}$ \\
\hline
\end{tabular}

Différence significative : $* \mathrm{p}<5 \% ; \mathrm{n}=5$

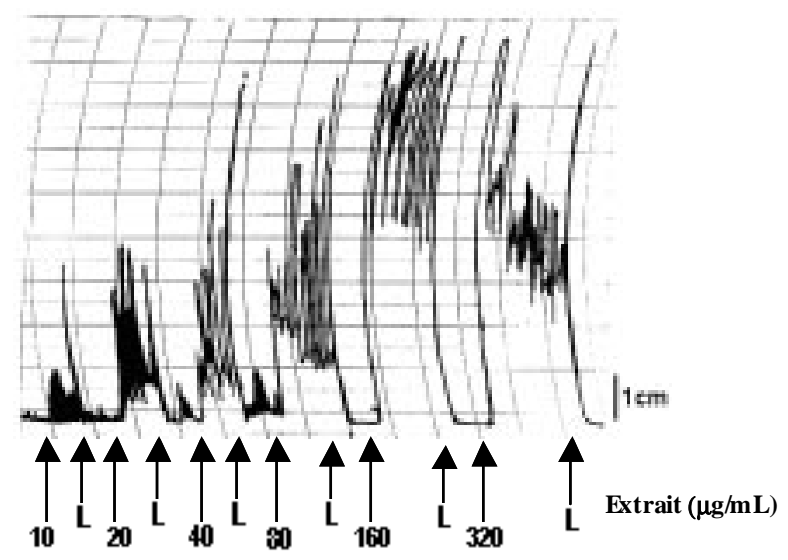

Figure 1 : Effets de l'extrait au cyclohexane de Bidens pilosa sur la contraction spontanée du muscle lisse intestinal de rat normal. ( $\mathrm{L}=$ lavage). 
En milieu physiologique dépourvu de calcium, l'augmentation du tonus de contraction (1046\%) induite par l'extrait de B. pilosa $(160 \mathrm{mg} / \mathrm{mL})$ est réduite d'environ $650 \%$ (fig. 2).

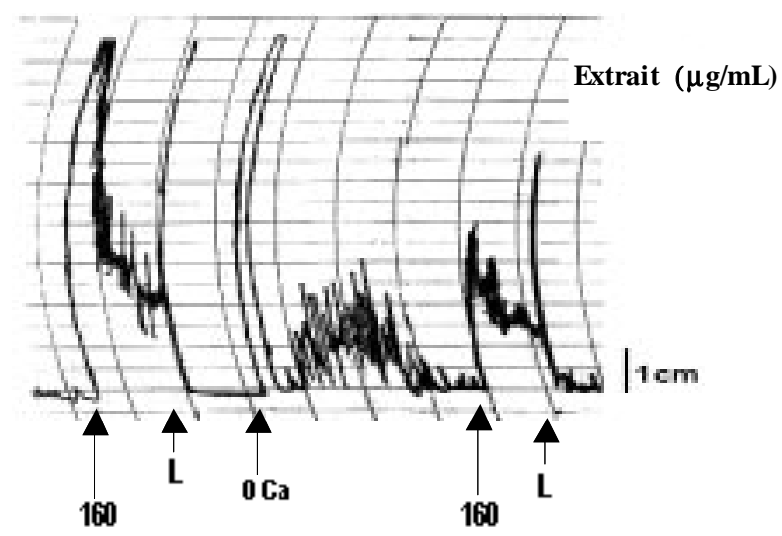

Figure 2 : Influence du milieu sans $\mathrm{Ca}^{++}(0 \mathrm{Ca})$ sur l'effet de l'extrait au cyclohexane de Bidens pilosa (B.p.) sur la contraction spontanée du muscle lisse intestinale du rat. ( $\mathrm{L}=$ lavage)

En milieu physiologique normale, le traitement préalable de l'iléon avec la nifédipine $(50 \mathrm{mg} / \mathrm{mL})$ inhibe totalement l'activité spasmogène de l'extrait (fig. 3).

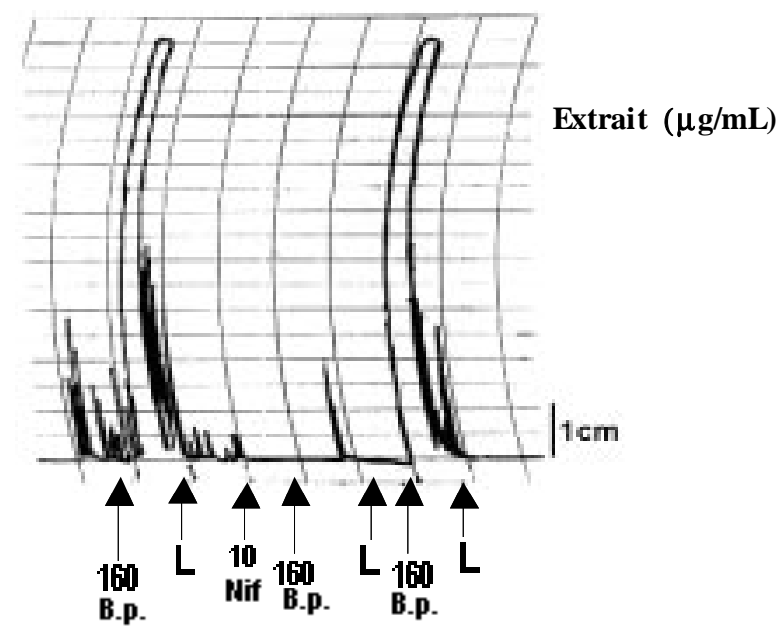

Figure 3 : Effet de la nifédipine (Nif) sur l'action de 'extrait au cyclohexane de Bidens pilosa (B.p.) sur la contraction spontanée du muscle lisse intestinale du rat. $(\mathrm{L}=$ lavage $)$

\section{DISCUSSION}

L'extrait de Bidens pilosa en milieu physiologique normal provoque au niveau du fragment d'iléon de rat, une augmentation de l'amplitude et du tonus de base des contractions. La contraction est augmentée à environ 1100
$\%$ de la valeur initiale. Cet effet stimulateur de l'extrait sur l'activité motrice de l'iléon pourrait évoquer quelques propriétés spasmogènes de $B$. pilosa ; ce qui expliquerait son utilisation dans le traitement de certains troubles digestifs, en l'occurrence la constipation (Castaigne et Lejonc, 1981). Les effets spasmogènes de l'extrait de $B$. pilosa et de l'acétylcholine sont inhibés respectivement de $77 \%$ et $94 \%$ par l'atropine. Ces résultats suggèrent une action de l'extrait de la plante par l'intermédiaire des récepteurs muscariniques. L'activation de ces récepteurs par l'ACh provoque une entrée d'ions $\mathrm{Ca}^{2+}$ due à une dépolarisation membranaire et est à l'origine de la contraction (Cloarec et al., 1989). Les résultats montrent qu'en présence d'un milieu sans calcium, B. pilosa induit un accroissement de la contraction iléale de $400 \%$ contre $1046 \%$ observée en milieu physiologique normal. L'augmentation de la contraction observée en milieu dépourvu de $\mathrm{Ca}^{2+}$ nous amène à penser que les ions calcium extracellulaire ne sont pas les seules sources activatrices. La mobilisation des ions $\mathrm{Ca}^{2+}$ des sites intracellulaires ne serait pas à exclure. L'extrait de $B$. pilosa agirait en augmentant l'échange $\mathrm{Na}^{+} / \mathrm{Ca}^{2+}$, mobilisant le $\mathrm{Ca}^{2+}$ intracellulaire. En effet, la nifédipine, substance connue pour son action bloquante des canaux calciques, inhibe totalement l'activité spasmogène de l'extrait. De ce fait, le mécanisme d'action de $B$. pilosa se situerait au niveau des canaux calciques. Ce résultat confirme en outre que dans le muscle lisse viscéral, la source première d'ions $\mathrm{Ca}^{2+}$ est le milieu extracellulaire.

\section{RÉFÉRENCESBIBLIOGRAPHIQUES}

Adjanohoun E.J.,Ake Assi L., Ali Ahmed, Eyme J., GuindoS., Kayonga A., Keita A. and Lebras M. (1988) Médecine traditionnelle et Pharmacopée. Contribution aux études botaniques et floristiques au Comores. Rapport Agence de Coopération Culturelle et Technique, Paris, p 243.

Amvam Zollo, Kuiate J.R., Menut C., Lamaty G., Bessiere J.M., Chalchat J.C. and Garry R.P. (1995) Aromatic plants of tropical central Africa. XX: The occurrence of 1-phenyl-1,3,5-triyne in the essential oil of Bidens pilosa $\mathrm{L}$. from Cameroun. Flavour and fragrance Journal ; 10, (2), 97 - 100.

Bouquet A. (1969) Féticheurs et médecine traditionnelle du Congo (Brazzaville). Mémoire ORSTOM, 36, 128p.

Brandao MG, Krettli A, Soares LS, Nery CG, Marinuzzi HC (1997) Antiamaial activity of extracts and fractions from Bidens pilosa and other Bidens species (Asteraceae) correlated with the presence of acetylene and flavonoid compounds. Journal of Ethnopharmacology, 57 (2), 131 - 138. 
Effets de l'extrait au cyclohexane de Bidens pilosa L. (asteraceae) sur la motricité intestinale de rat

Castaigne A and Lejonc JL (1981) Sémiologie médicale Initiation à la physiopathologie. Laboratoire Sandoz, pp $205-220$.

Cloarec D, Bruley Des Varannes S et Galmiche JP (1989) Modification de la motricité digestive. In Pharmacologie : Des concepts fondamentaux aux applications thérapeutiques. Ed. Frison - Roche, Paris, pp $581-589$.

Dimo T, Rakotonirina VS, Kamgang R, Tan PV, Kamanyi A and Bopelet M (1998) Effects of leaf aqueous extrait of Bidens pilosa (Asteraceae) on $\mathrm{KCl}$ - and norepinephrine-induced contractions of rat aorta. Journal of Ethnopharmacology, 60, 179 - 182.

Dimo T, Nguelefack TB, Kamtchouing P, Dongo E, Rakotonirina A et Rakotonirina SV (1999) Effets hypotensifs de l'extrait au méthanol de Bidens pilosa Linn chez les rats hypertendus. C.R. Académie des sciences, Paris, Sciences de la vie, 322, 323 - 329.

Hoffmann B and Holzi J (1989) Chalcones glycosides from Bidens pilosa. Phytochemistry, 28 (1), 247 - 249.

Kerharo J et Gadam J (19973) La pharmacopée sénégalaise traditionnelle - Plantes médicinales et toxiques. Ed. Vigot Frère, Paris, pp 220 - 223. 